\title{
Signed Product Cordial of the Sum and Union of Two Fourth Power of Paths and Cycles
}

\author{
Shokry Nada ${ }^{1}$, Amani Elrayes ${ }^{2}$, Ashraf Elrokh ${ }^{1}$, Aya Rabie $^{2}$ \\ ${ }^{1}$ Department of Mathematics, Faculty of Science, Menoyfia University, Cairo, Egypt \\ ${ }^{2}$ Institute of National Planning, Cairo, Egypt \\ Email address: \\ shokrynada@yahoo.com (S. Nada), amany.elrayes@inp.edu.eg (A. Elrayes), el-rokh@excite.co (A. Elrokh), \\ aya.ebrahim828@yahoo.com (A. Rabie)
}

\section{To cite this article:}

Shokry Nada, Amani Elrayes, Ashraf Elrokh, Aya Rabie. Signed Product Cordial of the Sum and Union of Two Fourth Power of Paths and Cycles. Machine Learning Research. Vol. 4, No. 4, 2019, pp. 45-50. doi: 10.11648/j.mlr.20190404.11

Received: June 15, 2019; Accepted: August 12, 2019; Published: December 30, 2019

\begin{abstract}
A simple graph is said to be signed product cordial if it admits \pm 1 labeling that satisfies certain conditions. Our aim in this paper is to contribute some new results on signed product cordial labeling and present necessary and sufficient conditions for signed product cordial of the sum and union of two fourth power of paths. We also study the signed product cordiality of the sum and union of fourth power cycles The residue classes modulo 4 are accustomed to find suitable labelings for each class to achieve our task. We have shown that the union and the join of any two fourth power of paths are always signed product cordial. Howover, the join and union of fourth power of cycles are only signed codial with some expectional situations.
\end{abstract}

Keywords: Fourth Power, Sum Graph, Union Graph, Signed Product Cordial Graph, AMS Classification: 05C78, 05C75, 05C20

\section{Introduction}

It is known that the graph theory and its branches have become interest topics for almost all fields of mathematics and also other area of science such as Chemistry, Biology, Physics, Communication, Economics, Engineering, Operator research and especially computer science $[1,3]$. We begin with finite, connected and undirected graph $G=(V, E)$ without loops and multiple edges. Here elements of sets $V(G)$ and $E(G)$ are known as vertices and edges respectively. The concept of graph labeling was introduced by Rosa in 1967 [9]. A graph labeling is an assignment of integers to the vertices or edges or both subject to certain condition (s). Most of the graph labeling problems have the following three common characteristics: a set of numbers for assignment of vertex labels, a rule that assigns a label to each edge and some condition (s) that these labels must satisfy. For detailed survey on graph labeling we refer to A Dynamic Survey of Graph Labeling by Gallian [8]. Cordial labeling was introduced by Cahit [2] who called a graph $G$ cordial if there is a vertex labeling $f: V(G) \rightarrow\{0,1\}$ such that the induced labeling $f^{*}: E(G) \rightarrow\{0,1\}$, defined by $f^{*}(x y)=|f(x)-f(y)|$, for all edges $x, y \in E(G)$ and with the following inequalities holding: $\left|v_{f}(0)-v_{f}(1)\right| \leq 1$ and $\left|e_{f}(0)-e_{f}(1)\right| \leq 1$, where $v_{f}(i)$ (respectively $e_{f}(i)$ ) is the number of vertices (respectively, edges) labeled with $i$. Motivated through the concept of cordial labeling the product cordial labeling was introduced by Sundaram et al [11] where absolute difference of vertex labels is replaced by product of vertex labels. Some researchers have written many papers in this field $[10,12]$.

In our work we test the signed product cordial of the sum of two fourth power of paths and cycles $P_{n}^{4}+P_{m}^{4}$ and $C_{n}^{4}+C_{m}^{4}$ and also the union of two fourth power of paths and cycles $P_{n}^{4} \cup P_{m}^{4}$ and $C_{n}^{4} \cup C_{m}^{4}$.

\section{Preliminaries}

Definition 1. A vertex labeling of graph $G f: V(G) \rightarrow$ $\{-1,1\}$ with induced edge labeling $f^{*}: E(G) \rightarrow\{-1,1\}$ defined by $f^{*}(u v)=f(u) \cdot f(v)$ is called a signed product cordial labeling if $\left|v_{f}(-1)-v_{f}(1)\right| \leq 1$ and $\mid e_{f}(-1)-$ $e_{f}(1) \mid \leq 1$, where $v_{f}(-1)$ is the number of vertices labeled with $-1, v_{f}(1)$ is the number of vertices labeled 1 , $e\left(f^{*}\right)(-1)$ is the number of edges labeled with -1 and 
$e\left(f^{*}\right)(1)$ is the number of edges labeled 1. A graph $G$ is signed product cordial if it admits signed product cordial labeling.

Definition 2. The Sum or join is $G_{1}+G_{2}$, is the graph with vertex set and edge set given by $V\left(G_{1}\right)+V\left(G_{2}\right)$ $=V\left(G_{1}\right) \cup V\left(G_{2}\right)$ and $E\left(G_{1}+G_{2}\right)=E\left(G_{1}\right) \cup E\left(G_{2}\right) \cup J$, where $J$ consists of edges join each vertex of $G_{1}$ to every vertex of $G_{2}$.

$$
\text { Definition } 3 . \quad \text { The union }
$$

Definition 4 . The fourth power of a cycles $C_{n}$ denoted by $C_{n}^{4}$ is $C_{n} \mathrm{UJ}$, where $\mathrm{J}$ is the set of all edges of the form edges $v_{i} v_{j}$ such that $2 \leq d\left(v_{i} v_{j}\right) \leq 4$ and $i<j$.

Diab [2-3] has reported several results concerning the sum and union of the cycles $C_{n}$ and paths $P_{m}$ together and with other specific graphs.

\section{Terminologies and Notations}

A cycle with $n$ vertices and $n$ edges is denoted by $C_{n}$, and its fourth power $C_{n}^{4}$ has $n$ vertices and $4 n-9$ edges. We let $L_{4 r}$ denote the labeling $(-1)_{2} 11$ $(-1)_{2} 11 \ldots(-1)_{2} 11$ (repeated $r$-times) and let $L_{4 r}^{\prime}$ denote the labeling $(-1) 11(-1) \quad(-1) 11(-1) \ldots(-1) 11(-1)$ (repeated $r$-times). The labeling $11(-1)_{2} 11(-1)_{2} \ldots 11(-1)_{2}$ (repeated $r$-times) and $1(-1)_{2} 11(-1)_{2} 1 \ldots 1(-1)_{2} 1$ (repeated $r$-times) are written $S_{4 r}$ and $S_{4 r}^{\prime}$ respectively. Let $M_{r}$ denote the labeling $(-1) 1(-1) 1 \ldots(-1) 1$, zero-one repeated $r$ times if $r$ is even and $(-1) 1(-1) 1 \ldots(-1) 1(-1)$ if $r$ is odd; for example, $\quad M_{6}=(-1) 1(-1) 1(-1) 1$ and $M_{5}=$ $(-1) 1(-1) 1(-1)$.

We let $M^{\prime}{ }_{r}$ denote the labeling $1(-1) 1(-1) \ldots 1(-1)$. Sometimes, we modify the labeling $M_{r}$ or $M_{r}^{\prime}$ by adding symbols at one end or the other (or both). Also, $L_{4 r}$ (or $L_{4 r}^{\prime}$ ) with extra labeling from right or left (or both sides). If $L$ is a labeling for fourth power of paths $P_{m}$ and $M$ is a labeling for fourth power of paths $P_{n}$, then we use the notation $[L ; M]$ to represent the labeling of the sum $P_{m}^{4}+P_{n}^{4}$. Additional notation that we use is the following. For a given labeling of the sum $P_{m}^{4}+P_{n}^{4}$, we let $v_{i}$ and $e_{i}$ (for $i=-1,1$ ) be the numbers of labels that are $i$ as before, we let $x_{i}$ and $a_{i}$ be the corresponding quantities for $P_{m}^{4}$, and we let $y_{i}$ and $b_{i}$ be those for $P_{n}^{4}$. It is easy to verify that $v_{-1}-v_{1}=\left(x_{-1}-x_{1}\right)+\left(y_{-1}-y_{1}\right)$ and $e_{-1}-e_{1}=\left(a_{-1}-a_{1}\right)+\left(b_{-1}-b_{1}\right)+\left(x_{-1}-x_{1}\right)\left(y_{-1}-\right.$ $\left.y_{1}\right)$.

Also for $P_{m}^{4} \cup P_{n}^{4}$, we use the same notation $[L ; M]$. Additional notation that we use is the following. For a given labeling of the union $P_{m}^{4} \cup P_{n}^{4}$, we let $v_{i}$ and $e_{i}$ (for $i=-1,1$ ) be the numbers of labels that are $i$ as before, we let $x_{i}$ and $a_{i}$ be the corresponding quantities for $P_{m}^{4}$, and we let $y_{i}$ and $b_{i}$ be those for $P_{n}^{4}$. It is easy to verify that $v_{-1}-v_{1}=$ $\left(x_{-1}-x_{1}\right)+\left(y_{-1}-y_{1}\right)$ and $e_{-1}-e_{1}=\left(a_{-1}-a_{1}\right)+$ $\left(b_{-1}-b_{1}\right)$. When it comes to the proof, we only need to show that, for each specified combination of labeling, $\left|v_{-1}-v_{1}\right| \leq$ 1 and $\left|e_{-1}-e_{1}\right| \leq 1$.

\section{Main Results}

\subsection{Signed Product Cordial of the Sum of Two Fourth Power of Paths}

In this subsection, we prove that the signed product cordial for the sum of two fourth power of paths. For this purpose let us study the following lemmas.

Lemma 4.1.1. If $n \equiv 0(\bmod 4)$, then $P_{n}^{4}+P_{m}^{4}$ is signed product cordial for all $m, n \geq 7$.

Proof. Suppose that $n=4 r$, where $r \geq 2$. The following cases will be examined.

Case $1 . m \equiv 0(\bmod 4)$.

Suppose that $m=4 s$, where $s \geq 2$. Then we label the vertices of $P_{4 r}^{4}+P_{4 s}^{4}$ by $\left[-1 L_{4 r-4}-111 ; 1_{2} L_{4 s-4}^{\prime}-1_{2}\right]$. Therefore $\quad x_{-1}=x_{1}=2 r, a_{-1}=a_{1}=8 r-5, y_{-1}=y_{1}=$ $2 s, b_{-1}=b_{1}=8 s-5$. It follows that $v_{-1}-v_{1}=\left(x_{-1}-\right.$ $\left.x_{1}\right)+\left(y_{-1}-y_{1}\right)=0$ and $e_{-1}-e_{1}=\left(a_{-1}-a_{1}\right)+\left(b_{-1}-\right.$ $\left.b_{1}\right)+\left(x_{-1}-x_{1}\right)\left(y_{-1}-y_{1}\right)=0$. As an example, Figure 1 illustrates $P_{8}^{4}+P_{8}^{4}$. Hence, $P_{4 r}^{4}+P_{4 s}^{4}$ is signed product cordial.

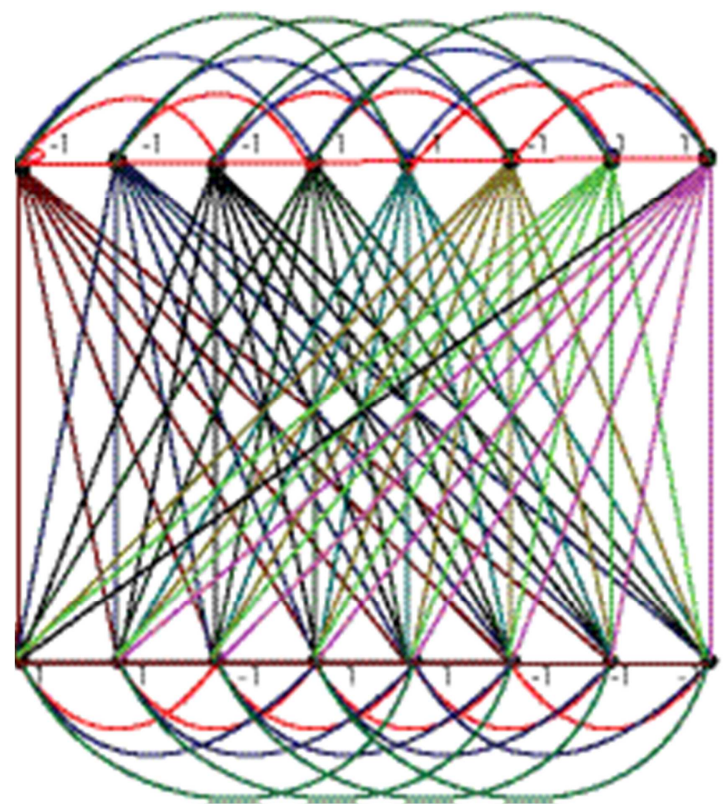

Figure 1. $P_{8}^{4}+P_{8}^{4}$.

Case $2 . m \equiv 1(\bmod 4)$.

Suppose that $m=4 s+1$, where $s \geq 2$. Then we label the vertices of $P_{4 r}^{4}+P_{4 s+1}^{4}$ by $\left[-1 L_{4 r-4}-111 ;-1_{2} L_{4 s-4} 1-\right.$ 11] . Therefore $x_{-1}=x_{1}=2 r, a_{-1}=a_{1}=8 r-5, y_{-1}=$ $2 s+1, y_{1}=2 s, b_{-1}=b_{1}=8 s-3$. It follows that $v_{-1}-$ $v_{1}=1$ and $e_{-1}-e_{1}=0$. Hence, $P_{4 r}^{4}+P_{4 s+1}^{4}$ is signed product cordial.

Case $3 . m \equiv 2(\bmod 4)$.

Suppose that $m=4 s+2$, where $s \geq 2$. Then we label the vertices of $P_{4 r}^{4}+P_{4 s+2}^{4}$ by $\left[-1 L_{4 r-4}-111 ;-11_{3}-\right.$ $\left.1 S_{4 s-4}-1\right]$. Therefore $x_{-1}=x_{1}=2 r, a_{-1}=a_{1}=8 r-$ $5, y_{-1}=y_{1}=2 s+1, b_{-1}=b_{1}=8 s-1$. It follows that $v_{-1}-v_{1}=0$ and $e_{-1}-e_{1}=0$. Hence, $P_{4 r}^{4}+P_{4 s+2}^{4}$ is signed product cordial.

Case $4 . m \equiv 3(\bmod 4)$. 
Suppose that $m=4 s+3$, where $s \geq 1$. Then we label the vertices of $P_{4 r}^{4}+P_{4 s+3}^{4}$ by $\left[-1 L_{4 r-4}-111 ;-1_{2} 1 L_{4 s}\right]$. Therefore $\quad x_{-1}=x_{1}=2 r, a_{-1}=a_{1}=8 r-5, y_{-1}=2 s+$ $2, y_{1}=2 s+1, b_{-1}=b_{1}=8 s+1$. It follows that $v_{-1}-$ $v_{1}=1$ and $e_{-1}-e_{1}=0$. Hence, $P_{4 r}^{4}+P_{4 s+3}^{4}$ is signed product cordial.

Lemma 4.1.2. If $n \equiv 1(\bmod 4)$, then $P_{n}^{4}+P_{m}^{4}$ is signed product cordial for all $m, n \geq 7$.

Proof. Suppose that $n=4 r+1$, where $r \geq 2$. The following cases will be examined.

Case $1 . m \equiv 1(\bmod 4)$.

Suppose that $m=4 s+1$, where $s \geq 2$. Then we label the vertices of $P_{4 r+1}^{4}+P_{4 s+1}^{4}$ by $\left[-1_{2} L_{4 r-4} 1-11 ; 1_{2} L_{4 s-4}^{\prime}-\right.$ $11-1]$. Therefore $x_{-1}=2 r+1, x_{1}=2 r, a_{-1}=a_{1}=$ $8 r-3, y_{-1}=2 s, y_{1}=2 s+1, b_{-1}=b_{1}=8 s-3$. It follows that $v_{-1}-v_{1}=0$ and $e_{-1}-e_{1}=1$. Hence, $P_{4 r+1}^{4}+P_{4 s+1}^{4}$ is signed product cordial.

Case $2 . m \equiv 2(\bmod 4)$.

Suppose that $m=4 s+2$, where $s \geq 2$. Then we label the vertices of $P_{4 r+1}^{4}+P_{4 s+2}^{4}$ by $\left[-1_{2} L_{4 r-4} 1-11 ;-11_{3}-\right.$ $\left.1 S_{4 s-4}-1\right]$. Therefore $x_{-1}=2 r+1, x_{1}=2 r, a_{-1}=a_{1}=$ $8 r-3, y_{-1}=y_{1}=2 s+1, b_{-1}=b_{1}=8 s-1$. It follows that $v_{-1}-v_{1}=1$ and $e_{-1}-e_{1}=0$. Hence, $P_{4 r+1}^{4}+P_{4 s+2}^{4}$ is signed product cordial.

Case $3 . m \equiv 3(\bmod 4)$.

Suppose that $m=4 s+3$, where $s \geq 1$. Then we label the vertices of $P_{4 r+1}^{4}+P_{4 s+3}^{4}$ by $\left[-1_{2} L_{4 r-4} 1-11 ; 1_{2} S_{4 s}-1\right]$. Therefore $\quad x_{-1}=2 r+1, x_{1}=2 r, a_{-1}=a_{1}=8 r-$ $3, y_{-1}=2 s+1, y_{1}=2 s+2, b_{-1}=b_{1}=8 s+1$. It follows that $v_{-1}-v_{1}=0$ and $e_{-1}-e_{1}=1$. Hence, $P_{4 r+1}^{4}+P_{4 s+3}^{4}$ is signed product cordial.

Lemma 4.1.3. If $n \equiv 2(\bmod 4)$, then $P_{n}^{4}+P_{m}^{4}$ is signed product cordial for all $m, n \geq 7$.

Proof. Suppose that $n=4 r+2$, where $r \geq 2$. The following cases will be examined.

Case $1 . m \equiv 2(\bmod 4)$.

Suppose that $m=4 s+2$, where $s \geq 2$. Then we label the vertices of $P_{4 r+2}^{4}+P_{4 s+2}^{4}$ by $\left[-11_{3}-1 S_{4 r-4}-1 ;-11_{3}-\right.$ $\left.1 S_{4 s-4}-1\right]$. Therefore $x_{-1}=x_{1}=2 r+1, a_{-1}=a_{1}=$ $8 r-1, y_{-1}=y_{1}=2 s+1, b_{-1}=b_{1}=8 s-1$. It follows that $v_{-1}-v_{1}=0$ and $e_{-1}-e_{1}=0$. Hence, $P_{4 r+2}^{4}+P_{4 s+2}^{4}$ is signed product cordial.

Case $2 . m \equiv 3(\bmod 4)$.

Suppose that $m=4 s+3$, where $s \geq 1$. Then we label the vertices of $P_{4 r+2}^{4}+P_{4 s+3}^{4}$ by $\left[-11_{3}-1 S_{4 r-4}-\right.$ $\left.1 ;-1_{2} 1 L_{4 s}\right]$. Therefore $x_{-1}=x_{1}=2 r+1, a_{-1}=a_{1}=$ $8 r-1, y_{-1}=2 s+2, y_{1}=2 s+1, b_{-1}=b_{1}=8 s+1$. It follows that $v_{-1}-v_{1}=1$ and $e_{-1}-e_{1}=0$. Hence, $P_{4 r+2}^{4}+P_{4 s+3}^{4}$ is signed product cordial.

Lemma 4.1 .4 . If $n \equiv 3(\bmod 4)$, then $P_{n}^{4}+P_{m}^{4}$ is signed product cordial for all $m, n \geq 7$.

Proof. Suppose that $n=4 r+3$, where $r \geq 2$. The following cases will be examined.

Case $1 . m \equiv 3(\bmod 4)$.

Suppose that $m=4 s+3$, where $s \geq 1$. Then we label the vertices of $P_{4 r+3}^{4}+P_{4 s+3}^{4}$ by $\left[-1_{2} 1 L_{4 r} ; 1_{2} S_{4 s}-1\right]$. Therefore $\quad x_{-1}=2 r+2, x_{1}=2 r+1, a_{-1}=a_{1}=8 r+$ $1, y_{-1}=2 s+1, y_{1}=2 s+2, b_{-1}=b_{1}=8 s+1$. It follows that $v_{-1}-v_{1}=0$ and $e_{-1}-e_{1}=-1$. Hence, $P_{4 r+3}^{4}+$ $P_{4 s+3}^{4}$ is signed product cordial.

As a consequence of all lemmas mentioned above we conclude that

Theorem 4.1 The sum of two fourth Power of Paths $P_{n}^{4}+P_{m}^{4}$ is signed product cordial for all $m, n \geq 7$.

\subsection{Signed Product Cordial of Sum of Two Fourth Power of Cycles}

Here, we study The signed product cordial of sum of two fourth Power of cycles.

Lemma 4.2.1. If $n \equiv 0(\bmod 4)$, then $C_{n}^{4}+C_{m}^{4}$ is signed product cordial for all $m, n \geq 7$.

Proof. Suppose that $n=4 r$, where $r \geq 2$. The following cases will be examined.

Case $1 . m \equiv 0(\bmod 4)$.

Suppose that $m=4 s$, where $s \geq 2$. Then we label the vertices of $C_{4 r}^{4}+C_{4 s}^{4} \quad$ by $\left[S^{\prime}{ }_{4 r} ; 1_{3} M_{4 s-6}-1_{3}\right]$ Therefore $x_{-1}=x_{1}=2 r, a_{-1}=8 r-$ $4, a_{1}=8 r-5, y_{-1}=y_{1}=2 s, b_{-1}=8 s-5, b_{1}=8 s-4$. It follows that $v_{-1}-v_{1}=\left(x_{-1}-x_{1}\right)+\left(y_{-1}-y_{1}\right)=0$ and $e_{-1}-e_{1}=\left(a_{-1}-a_{1}\right)+\left(b_{-1}-b_{1}\right)+\left(x_{-1}-x_{1}\right)\left(y_{-1}-\right.$ $\left.y_{1}\right)=0$. Hence, $C_{4 r}^{4}+C_{4 s}^{4}$ is signed product cordial.

Case $2 . m \equiv 1(\bmod 4)$.

Suppose that $m=4 s+1$, where $s \geq 2$. Then we label the vertices of $C_{4 r}^{4}+C_{4 s+1}^{4}$ by $\left[1_{3} M_{4 r-6}-1_{3} ; L_{4 s}-1\right]$. Therefore $\quad x_{-1}=x_{1}=2 r, a_{-1}=8 r-5, a_{1}=8 r-$ $4, y_{-1}=2 s+1, y_{1}=2 s, b_{-1}=8 s-2, b_{1}=8 s-3$. It follows that $v_{-1}-v_{1}=1$ and $e_{-1}-e_{1}=0$. Hence, $C_{4 r}^{4}+C_{4 s+1}^{4}$ is signed product cordial.

Case $3 . m \equiv 2(\bmod 4)$.

Suppose that $m=4 s+2$, where $s \geq 2$. Then we label the vertices of $C_{4 r}^{4}+C_{4 s+2}^{4}$ by $\left[S^{\prime}{ }_{4 r} ;-1_{3} 1-11_{3} M_{4 s-6}\right]$. Therefore $\quad x_{-1}=x_{1}=2 r, a_{-1}=8 r-4, a_{1}=8 r-$ $5, y_{-1}=y_{1}=2 s+1, b_{-1}=8 s-1, b_{1}=8 s$. It follows that $v_{-1}-v_{1}=0$ and $e_{-1}-e_{1}=0$. Hence, $C_{4 r}^{4}+C_{4 s+2}^{4}$ is signed product cordial.

Case $4 . m \equiv 3(\bmod 4)$.

Suppose that $m=4 s+3$, where $s \geq 1$. Then we label the vertices of $C_{4 r}^{4}+C_{4 s+3}^{4}$ by $\left[1_{3} M_{4 r-6}-1_{3} ; L_{4 s}^{\prime}-11-1\right]$. Therefore $\quad x_{-1}=x_{1}=2 r, a_{-1}=8 r-5, a_{1}=8 r-$ $4, y_{-1}=2 s+2, y_{1}=2 s+1, b_{-1}=8 s+2, b_{1}=8 s+1$. It follows that $v_{-1}-v_{1}=1$ and $e_{-1}-e_{1}=0$. Hence, $C_{4 r}^{4}+C_{4 s+3}^{4}$ is signed product cordial.

Lemma 4.2 .2 . If $n \equiv 1(\bmod 4)$, then $C_{n}^{4}+C_{m}^{4}$ is signed product cordial for all $m, n \geq 7$.

Proof. Suppose that $n=4 r+1$, where $r \geq 2$. The following cases will be examined.

Case $1 . m \equiv 1(\bmod 4)$.

Suppose that $m=4 s+1$, where $s \geq 2$. Then we label the vertices of $C_{4 r+1}^{4}+C_{4 s+1}^{4}$ by $\left[L_{4 r}-1 ; 1_{3} L_{4 s-4}^{\prime}-1_{2}\right]$. Therefore $x_{-1}=2 r+1, x_{1}=2 r, a_{-1}=8 r-2, a_{1}=8 r-$ $3, y_{-1}=2 s, y_{1}=2 s+1, b_{-1}=8 s-3, b_{1}=8 s-2$. It follows that $v_{-1}-v_{1}=0$ and $e_{-1}-e_{1}=1$. Hence, $C_{4 r+1}^{4}+C_{4 s+1}^{4}$ is signed product cordial.

Case $2 . m \equiv 2(\bmod 4)$.

Suppose that $m=4 s+2$, where $s \geq 2$. Then we label the vertices of $C_{4 r+1}^{4}+C_{4 s+2}^{4}$ by $\left[L_{4 r}-1 ;-1_{3} 1-11_{3} M_{4 s-6}\right]$. 
Therefore $x_{-1}=2 r+1, x_{1}=2 r, a_{-1}=8 r-2, a_{1}=8 r-$ $3, y_{-1}=y_{1}=2 s+1, b_{-1}=8 s-1, b_{1}=8 s$. It follows that $v_{-1}-v_{1}=1$ and $e_{-1}-e_{1}=0$. Hence, $C_{4 r+1}^{4}+C_{4 s+2}^{4}$ is signed product cordial.

Case $3 . m \equiv 3(\bmod 4)$.

Suppose that $m=4 s+3$, where $s \geq 1$. Then we label the vertices of $C_{4 r+1}^{4}+C_{4 s+3}^{4}$ by $\left[1_{3} L_{4 r-4}^{\prime}-1_{2} ; L_{4 s}^{\prime}-11-1\right]$. Therefore $x_{-1}=2 r, x_{1}=2 r+1, a_{-1}=8 r-3, a_{1}=8 r-$ $2, y_{-1}=2 s+2, y_{1}=2 s+1, b_{-1}=8 s+2, b_{1}=8 s+1$. It follows that $v_{-1}-v_{1}=0$ and $e_{-1}-e_{1}=1$. Hence, $C_{4 r+1}^{4}+C_{4 s+3}^{4}$ is signed product cordial.

Lemma 4.2 .3 . If $n \equiv 2(\bmod 4)$, then $C_{n}^{4}+C_{m}^{4}$ is signed product cordial for all $m, n \geq 7$.

Proof. Suppose that $n=4 r+2$, where $r \geq 2$. The following cases will be examined.

Case $1 . m \equiv 2(\bmod 4)$.

Suppose that $m=4 s+2$, where $s \geq 2$. Then we label the vertices of $C_{4 r+2}^{4}+C_{4 s+2}^{4}$ by $\left[-1_{3} 1_{3} L_{4 r-4}^{\prime} ;-1_{3} 1-\right.$ $\left.11_{3} M_{4 s-6}\right]$. Therefore $x_{-1}=x_{1}=2 r+1, a_{-1}=8 r, a_{1}=$ $8 r-1, y_{-1}=y_{1}=2 s+1, b_{-1}=8 s-1, b_{1}=8 s$ follows that $v_{-1}-v_{1}=0$ and $e_{-1}-e_{1}=0$. Hence, $C_{4 r+2}^{4}+C_{4 s+2}^{4}$ is signed product cordial.

Case $2 . m \equiv 3(\bmod 4)$.

Suppose that $m=4 s+3$, where $s \geq 1$. Then we label the vertices of $C_{4 r+2}^{4}+C_{4 s+3}^{4}$ by $\left[-1_{3} 1-11_{3} M_{4 s-6} ; L_{4 s}^{\prime}-\right.$ $11-1]$ Therefore $x_{-1}=x_{1}=2 r+1, a_{-1}=8 r-1, a_{1}=$ $8 r, y_{-1}=2 s+2, y_{1}=2 s+1, b_{-1}=8 s+2, b_{1}=8 s+1$. It follows that $v_{-1}-v_{1}=1$ and $e_{-1}-e_{1}=0$. Hence, $C_{4 r+2}^{4}+C_{4 s+3}^{4}$ is signed product cordial.

As a consequence of all lemmas mentioned in subsection 4.2 we conclude that

Theorem 4.1. The sum of two fourth Power of cycles $C_{n}^{4}+C_{m}^{4}$ is signed product cordial for all $m, n \geq 7$ except at $(m, n)=(7,7)$

4.3 Signed product cordial of union of two fourth Power of Paths

Here, we prove that signed product cordial of the union of two fourth Power of Paths. To achieve this result, let us study the following lemmas.

Lemma 4.3.1. If $n \equiv 0(\bmod 4)$, then $P_{n}^{4} \cup P_{m}^{4}$ is signed product cordial for all $m, n \geq 7$.

Proof. Suppose that $n=4 r$, where $r \geq 2$. The following cases will be examined.

Case $1 . m \equiv 0(\bmod 4)$.

Suppose that $m=4 s$, where $s \geq 2$. Then we label the vertices of $P_{4 r}^{4} \cup P_{4 s}^{4}$ by $\left[-1 L_{4 r-4}-111 ; 1_{2} L_{4 s-4}^{\prime}-1_{2},\right]$. Therefore $\quad x_{-1}=x_{1}=2 r, a_{-1}=a_{1}=8 r-5, y_{-1}=y_{1}=$ $2 s, b_{-1}=b_{1}=8 s-5$. It follows that $v_{-1}-v_{1}=\left(x_{-1}-\right.$ $\left.x_{1}\right)+\left(y_{-1}-y_{1}\right)=0$ and $e_{-1}-e_{1}=\left(a_{-1}-a_{1}\right)+\left(b_{-1}-\right.$ $\left.b_{1}\right)=0$. As an example, Figure 2 illustrates $P_{8}^{4} \cup P_{8}^{4}$. Hence, $P_{4 r}^{4} \cup P_{4 s}^{4}$ is signed product cordial.

Case $2 . m \equiv 1(\bmod 4)$.

Suppose that $m=4 s+1$, where $s \geq 2$. Then we label the vertices of $P_{4 r}^{4} \cup P_{4 s+1}^{4}$ by $\left[-1 L_{4 r-4}-111 ;-1_{2} L_{4 s-4} 1-\right.$ 11] . Therefore $x_{-1}=x_{1}=2 r, a_{-1}=a_{1}=8 r-5, y_{-1}=$ $2 s+1, y_{1}=2 s, b_{-1}=b_{1}=8 s-3$. It follows that $v_{-1}-$ $v_{1}=1$ and $e_{-1}-e_{1}=0$. Hence, $P_{4 r}^{4} \cup P_{4 s+1}^{4}$ is signed product cordial.
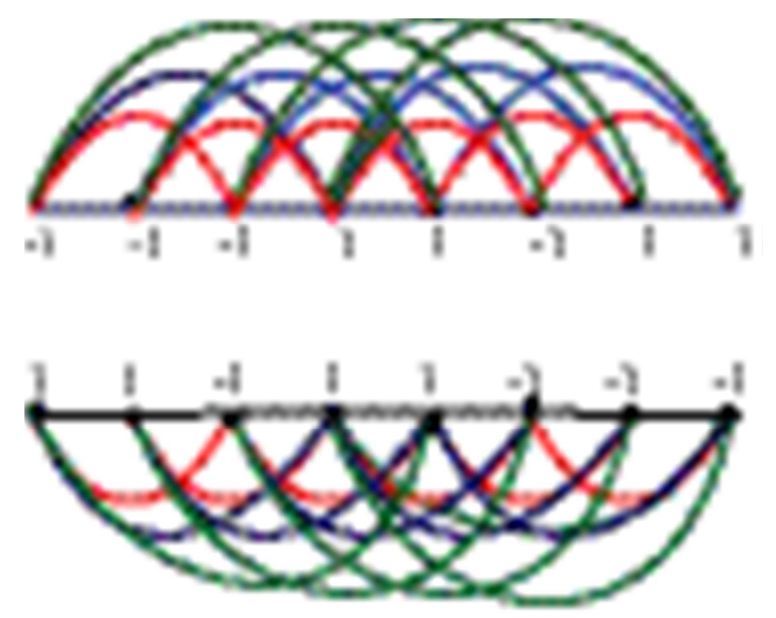

Figure 2. $P_{8}^{4} \cup P_{8}^{4}$.

Case $3 . m \equiv 2(\bmod 4)$.

Suppose that $m=4 s+2$, where $s \geq 2$. Then we label the vertices of $P_{4 r}^{4} \cup P_{4 s+2}^{4}$ by $\left[-1 L_{4 r-4}-111 ;-11_{3}-\right.$ $\left.1 S_{4 s-4}-1\right]$. Therefore $x_{-1}=x_{1}=2 r, a_{-1}=a_{1}=8 r-$ $5, y_{-1}=y_{1}=2 s+1, b_{-1}=b_{1}=8 s-1$. It follows that $v_{-1}-v_{1}=0$ and $e_{-1}-e_{1}=0$. Hence, $P_{4 r}^{4} \cup P_{4 s+2}^{4}$ is signed product cordial.

Case $4 . m \equiv 3(\bmod 4)$.

Suppose that $m=4 s+3$, where $s \geq 1$. Then we label the vertices of $P_{4 r}^{4} \cup P_{4 s+3}^{4}$ by $\left[-1 L_{4 r-4}-111 ;-1_{2} 1 L_{4 s}\right]$. Therefore $\quad x_{-1}=x_{1}=2 r, a_{-1}=a_{1}=8 r-5, y_{-1}=2 s+$ $2, y_{1}=2 s+1, b_{-1}=b_{1}=8 s+1$. It follows that $v_{-1}-$ $v_{1}=1$ and $e_{-1}-e_{1}=0$. Hence, $P_{4 r}^{4} \cup P_{4 s+3}^{4}$ is signed product cordial.

Lemma 4.3.2. If $n \equiv 1(\bmod 4)$, then $P_{n}^{4} \cup P_{m}^{4}$ is signed product cordial for all $m, n \geq 7$.

Proof. Suppose that $n=4 r+1$, where $r \geq 2$. The following cases will be examined.

Case $1 . m \equiv 1(\bmod 4)$.

Suppose that $m=4 s+1$, where $s \geq 2$. Then we label the vertices of $P_{4 r+1}^{4} \cup P_{4 s+1}^{4}$ by $\left[-1_{2} L_{4 r-4} 1-11 ; 1_{2} L_{4 s-4}^{\prime}-\right.$ $11-1]$. Therefore $x_{-1}=2 r+1, x_{1}=2 r, a_{-1}=a_{1}=$ $8 r-3, y_{-1}=2 s, y_{1}=2 s+1, b_{-1}=b_{1}=8 s-3$. It follows that $v_{-1}-v_{1}=0$ and $e_{-1}-e_{1}=0$. Hence, $P_{4 r+1}^{4} \cup P_{4 s+1}^{4}$ is signed product cordial.

Case $2 . m \equiv 2(\bmod 4)$.

Suppose that $m=4 s+2$, where $s \geq 2$. Then we label the vertices of $P_{4 r+1}^{4} \cup P_{4 s+2}^{4}$ by $\left[-1_{2} L_{4 r-4} 1-11 ;-11_{3}-\right.$ $\left.1 S_{4 s-4}-1\right]$. Therefore $x_{-1}=2 r+1, x_{1}=2 r, a_{-1}=a_{1}=$ $8 r-3, y_{-1}=y_{1}=2 s+1, b_{-1}=b_{1}=8 s-1$. It follows that $v_{-1}-v_{1}=1$ and $e_{-1}-e_{1}=0$. Hence, $P_{4 r+1}^{4} \cup P_{4 s+2}^{4}$ is signed product cordial.

Case $3 . m \equiv 3(\bmod 4)$.

Suppose that $m=4 s+3$, where $s \geq 1$. Then we label the vertices of $P_{4 r+1}^{4} \cup P_{4 s+3}^{4}$ by $\left[-1_{2} L_{4 r-4} 1-11 ; 1_{2} S_{4 s}-1\right]$. Therefore $\quad x_{-1}=2 r+1, x_{1}=2 r, a_{-1}=a_{1}=8 r-$ $3, y_{-1}=2 s+1, y_{1}=2 s+2, b_{-1}=b_{1}=8 s+1$. It follows that $v_{-1}-v_{1}=0$ and $e_{-1}-e_{1}=0$. Hence, $P_{4 r+1}^{4} \cup P_{4 s+3}^{4}$ is signed product cordial.

Lemma 4.3.3. If $n \equiv 2(\bmod 4)$, then $P_{n}^{4} \cup P_{m}^{4}$ is signed product cordial for all $m, n \geq 7$. 
Proof. Suppose that $n=4 r+2$, where $r \geq 2$. The following cases will be examined.

Case $1 . m \equiv 2(\bmod 4)$.

Suppose that $m=4 s+2$, where $s \geq 2$. Then we label the vertices of $P_{4 r+2}^{4} \cup P_{4 s+2}^{4}$ by $\left[-11_{3}-1 S_{4 r-4}-1 ;-11_{3}-\right.$ $\left.1 S_{4 s-4}-1\right]$. Therefore $x_{-1}=x_{1}=2 r+1, a_{-1}=a_{1}=$ $8 r-1, y_{-1}=y_{1}=2 s+1, b_{-1}=b_{1}=8 s-1$. It follows that $v_{-1}-v_{1}=0$ and $e_{-1}-e_{1}=0$. Hence, $P_{4 r+2}^{4} \cup P_{4 s+2}^{4}$ is signed product cordial.

Case $2 . m \equiv 3(\bmod 4)$.

Suppose that $m=4 s+3$, where $s \geq 1$. Then we label the vertices of $P_{4 r+2}^{4} \cup P_{4 s+3}^{4}$ by $\left[-11_{3}-1 S_{4 r-4}-1 ;-1_{2} 1 L_{4 s}\right]$ Therefore $\quad x_{-1}=x_{1}=2 r+1, a_{-1}=a_{1}=8 r-1, y_{-1}=$ $2 s+2, y_{1}=2 s+1, b_{-1}=b_{1}=8 s+1$. It follows that $v_{-1}-v_{1}=1$ and $e_{-1}-e_{1}=0$. Hence, $P_{4 r+2}^{4} \cup P_{4 s+3}^{4}$ is signed product cordial.

Lemma 4.3.4. If $n \equiv 3(\bmod 4)$, then $P_{n}^{4} \cup P_{m}^{4}$ is signed product cordial for all $m, n \geq 7$.

Proof. Suppose that $n=4 r+3$, where $r \geq 2$. The following cases will be examined.

Case $1 . m \equiv 3(\bmod 4)$.

Suppose that $m=4 s+3$, where $s \geq 1$. Then we label the vertices of $P_{4 r+3}^{4} \cup P_{4 s+3}^{4}$ by $\left[-1_{2} 1 L_{4 r} ; 1_{2} S_{4 s}-1\right]$. Therefore $\quad x_{-1}=2 r+2, x_{1}=2 r+1, a_{-1}=a_{1}=8 r+$ $1, y_{-1}=2 s+1, y_{1}=2 s+2, b_{-1}=b_{1}=8 s+1$. It follows that $v_{-1}-v_{1}=0$ and $e_{-1}-e_{1}=0$. Hence, $P_{4 r+3}^{4} \cup P_{4 s+3}^{4}$ is signed product cordial.

As a consequence of all lemmas proved in subsection 4.3 we conclude that

Theorem 4.3 The union of two fourth Power of Paths $P_{n}^{4} \cup P_{m}^{4}$ is signed product cordial for all $m, n \geq 7$.

4.4 Signed product cordial of union of two fourth Power of cycles

Here, we prove that the signed product cordial of the union of two fourth Power of cycles. For this purpose let us study the following lemmas.

Lemma 4.4.1. If $n \equiv 0(\bmod 4)$, then $C_{n}^{4} \cup C_{m}^{4}$ is signed product cordial for all $m, n \geq 7$.

Proof. Suppose that $n=4 r$, where $r \geq 2$. The following cases will be examined.

Case $1 . m \equiv 0(\bmod 4)$.

Suppose that $m=4 s$, where $s \geq 2$. Then we label the vertices of $C_{4 r}^{4} \cup C_{4 s}^{4}$ by $\left[S^{\prime}{ }_{4 r} ; 1_{3} M_{4 s-6}-1_{3}\right]$. Therefore $x_{-1}=x_{1}=2 r, a_{-1}=8 r-4, a_{1}=8 r-5, y_{-1}=y_{1}=$

$2 s, b_{-1}=8 s-5, b_{1}=8 s-4$. It follows that $v_{-1}-v_{1}=$ $\left(x_{-1}-x_{1}\right)+\left(y_{-1}-y_{1}\right)=0$ and $e_{-1}-e_{1}=\left(a_{-1}-a_{1}\right)+$ $\left(b_{-1}-b_{1}\right)=0$. Hence, $C_{4 r}^{4} \cup C_{4 s}^{4}$ is signed product cordial.

Case $2 . m \equiv 1(\bmod 4)$.

Suppose that $m=4 s+1$, where $s \geq 2$. Then we label the vertices of $C_{4 r}^{4} \cup C_{4 s+1}^{4}$ by $\left[1_{3} M_{4 r-6}-1_{3} ; L_{4 s}-1\right]$. Therefore $\quad x_{-1}=x_{1}=2 r, a_{-1}=8 r-5, a_{1}=8 r-$ $4, y_{-1}=2 s+1, y_{1}=2 s, b_{-1}=8 s-2, b_{1}=8 s-3$. It follows that $v_{-1}-v_{1}=1$ and $e_{-1}-e_{1}=0$. Hence, $C_{4 r}^{4} \cup C_{4 s+1}^{4}$ is signed product cordial.

Case $3 . m \equiv 2(\bmod 4)$.

Suppose that $m=4 s+2$, where $s \geq 2$. Then we label the vertices of $C_{4 r}^{4} \cup C_{4 s+2}^{4}$ by $\left[S^{\prime}{ }_{4 r} ; 31-11_{3} M_{4 s-6}\right]$. Therefore $x_{-1}=x_{1}=2 r, a_{-1}=8 r-4, a_{1}=8 r-5, y_{-1}=y_{1}=$
$2 s+1, b_{-1}=8 s-1, b_{1}=8 s$. It follows that $v_{-1}-v_{1}=0$ and $e_{-1}-e_{1}=0$. Hence, $C_{4 r}^{4} \cup C_{4 s+2}^{4}$ is signed product cordial.

Case $4 . m \equiv 3(\bmod 4)$.

Suppose that $m=4 s+3$, where $s \geq 1$. Then we label the vertices of $C_{4 r}^{4} \cup C_{4 s+3}^{4}$ by $\left[1_{3} M_{4 r-6}-1_{3} ; L_{4 s}^{\prime}-11-1\right]$. Therefore $\quad x_{-1}=x_{1}=2 r, a_{-1}=8 r-5, a_{1}=8 r-$ $4, y_{-1}=2 s+2, y_{1}=2 s+1, b_{-1}=8 s+2, b_{1}=8 s+1$. It follows that $v_{-1}-v_{1}=1$ and $e_{-1}-e_{1}=0$. Hence, $C_{4 r}^{4} \cup C_{4 s+3}^{4}$ is signed product cordial.

Lemma 4.4.2. If $n \equiv 1(\bmod 4)$, then $C_{n}^{4} \cup C_{m}^{4}$ is signed product cordial for all $m, n \geq 7$.

Proof. Suppose that $n=4 r+1$, where $r \geq 2$. The following cases will be examined.

Case $1 . m \equiv 1(\bmod 4)$.

Suppose that $m=4 s+1$, where $s \geq 2$. Then we label the vertices of $C_{4 r+1}^{4} \cup C_{4 s+1}^{4}$ by $\left[L_{4 r}-1 ; 1_{3} L_{4 s-4}^{\prime}-1_{2}\right]$. Therefore $x_{-1}=2 r+1, x_{1}=2 r, a_{-1}=8 r-2, a_{1}=8 r-$ $3, y_{-1}=2 s, y_{1}=2 s+1, b_{-1}=8 s-3, b_{1}=8 s-2$. It follows that $v_{-1}-v_{1}=0$ and $e_{-1}-e_{1}=0$. Hence, $C_{4 r+1}^{4} \cup C_{4 s+1}^{4}$ is signed product cordial.

Case $2 . m \equiv 2(\bmod 4)$.

Suppose that $m=4 s+2$, where $s \geq 2$. Then we label the vertices of $C_{4 r+1}^{4} \cup C_{4 s+2}^{4}$ by $\left[L_{4 r}-1 ;-1_{3} 1-11_{3} M_{4 s-6}\right]$. Therefore $x_{-1}=2 r+1, x_{1}=2 r, a_{-1}=8 r-2, a_{1}=8 r-$ $3, y_{-1}=y_{1}=2 s+1, b_{-1}=8 s-1, b_{1}=8 s$. It follows that $v_{-1}-v_{1}=1$ and $e_{-1}-e_{1}=0$. Hence, $C_{4 r+1}^{4} \cup C_{4 s+2}^{4}$ is signed product cordial.

Case $3 . m \equiv 3(\bmod 4)$.

Suppose that $m=4 s+3$, where $s \geq 1$. Then we label the vertices of $C_{4 r+1}^{4} \cup C_{4 s+3}^{4}$ by $\left[1_{3} L^{\prime}{ }_{4 r-4}-1_{2} ; L^{\prime}{ }_{4 s}-11-1\right]$ Therefore $x_{-1}=2 r, x_{1}=2 r+1, a_{-1}=8 r-3, a_{1}=8 r-$ $2, y_{-1}=2 s+2, y_{1}=2 s+1, b_{-1}=8 s+2, b_{1}=8 s+1$. It follows that $v_{-1}-v_{1}=0$ and $e_{-1}-e_{1}=0$. Hence, $C_{4 r+1}^{4} \cup C_{4 s+3}^{4}$ is signed product cordial.

Lemma 4.4.3. If $n \equiv 2(\bmod 4)$, then $C_{n}^{4} \cup C_{m}^{4}$ is signed product cordial for all $m, n \geq 7$.

Proof. Suppose that $n=4 r+2$, where $r \geq 2$. The following cases will be examined.

Case $1 . m \equiv 2(\bmod 4)$.

Suppose that $m=4 s+2$, where $s \geq 2$. Then we label the vertices of $C_{4 r+2}^{4} \cup C_{4 s+2}^{4}$ by $\left[-1_{3} 1_{3} L_{4 r-4}^{\prime} ;-1_{3} 1-\right.$ $\left.11_{3} M_{4 s-6}\right]$. Therefore $x_{-1}=x_{1}=2 r+1, a_{-1}=8 r, a_{1}=$ $8 r-1, y_{-1}=y_{1}=2 s+1, b_{-1}=8 s-1, b_{1}=8 s \quad$. It follows that $v_{-1}-v_{1}=0$ and $e_{-1}-e_{1}=0$. Hence, $C_{4 r+2}^{4} \cup C_{4 s+2}^{4}$ is signed product cordial.

Case $2 . m \equiv 3(\bmod 4)$.

Suppose that $m=4 s+3$, where $s \geq 1$. Then we label the vertices of $C_{4 r+2}^{4} \cup C_{4 s+3}^{4}$ by $\left[-1_{3} 1-11_{3} M_{4 s-6} ; L_{4 s}^{\prime}-\right.$ $11-1]$. Therefore $x_{-1}=x_{1}=2 r+1, a_{-1}=8 r-1, a_{1}=$ $8 r, y_{-1}=2 s+2, y_{1}=2 s+1, b_{-1}=8 s+2, b_{1}=8 s+1$. It follows that $v_{-1}-v_{1}=1$ and $e_{-1}-e_{1}=0$. Hence, $C_{4 r+2}^{4} \cup C_{4 s+3}^{4}$ is signed product cordial.

As a consequence of all lemmas proved in subsection 4.4 we conclude that.

Theorem 4.4 The union of two fourth Power of Paths $C_{n}^{4} \cup C_{m}^{4}$ is signed product cordial for all $m, n \geq 7$ except at $(n, m)=(7,7)$. 


\section{Conclusion}

In this paper we test the signed product cordial of the sum and union of two fourth power of paths and also for cycles, we found that $P_{n}^{4}+P_{m}^{4}$ and $P_{n}^{4} \cup P_{m}^{4}$ is signed product cordial for all $m, n$, and also $C_{n}^{4}+C_{m}^{4}$ and $C_{n}^{4} \cup C_{m}^{4}$ is signed product cordial for all $m, n$ except at $(n, m)=(7,7)$.

In our next work we will continue studying the signed product cordial of the sum and union of high powers of paths, cycles and other graphs.

\section{Acknowledgements}

The authors are thankful to the anonymous referee for useful suggestions and valuable comments.

\section{References}

[1] A. Balaban, "Application of Graph Theory in Chemistry", Journal of Chemical Information and Computer Science, 334-343 (2019).

[2] I. Cahit, Cordial Graphs. A weaker version of graceful and harmonious Graphs, Ars Combinatoria, 23: 201-207 (1987).

[3] A. Chakraborty, T. Dutta, S. Mondal, and A. Nath, "Application of Graph Theory in Social Media", International Journal of Computer Sciences and Engineering, vol. -6, 722-729 (2018).
[4] J. Devaraj and P. Delphy. On signed cordial graph, Int. J. of Mathematical Sciences and Applications, Vol. 1, No. 3, September 1156-1167 (2011).

[5] A. T. Diab, Study of some problems of cordial graphs, Ars Combin, 92, 255-261 (2009).

[6] A. T. Diab, Generalization of some results on cordial graphs, Ars Combin, 99, 161-173 (2011).

[7] F. Farahani, W. Karwski, and N. R. Lighthall, "Application of Graph Theory for Identifing Connectivity Patterns in Human Brain Network: A S ystematic Review", frontiers in Neuroscience, (2019).

[8] J. A. Gallian, A Dynamic Survey of Graph Labeling, The Electronic Journal of Combinatorics, DS6, (2014).

[9] A. Rosa, On certain valuations of the vertices of a graph, Theory of graphs (Internat. Symposium, Rome, July 1996), Gordon and Breach, N. Y. and Dunod Paris 349- 355, (1967).

[10] P. Sumathi, A. Mahalakshmi, "Quotient-3 Cordial Labeling for Path Related Graphs", Applied Mathematics and Scientific Computing, 555-561 (2019).

[11] M. Sundaram, R. Ponraj, and S. Somasundaram, Product cordial labeling of graph, Bulletin of Pure and Applied Science, vol. 23, 155-163, (2004).

[12] R. Ponraj, K. Annathurai and R. Kala, "Remainder Cordiality of some Graphs", Palestine Journal of Mathematics, Vol. 8 (1), 367-372 (2019). 